RENNIE ON PHLORIZIN.

LXI.-On Phlorizin.

By Edward H. Rennie, M.A., D.Sc., Professor of Chemistry in the University of Adelaide, South Australia.

Is a paper on "Glycyphyllin, the Sweet Principle of Smilax Glycyphylla," commanicated to the Society some few months ago (Trans., 1886,857 ), it was suggested as possible, though scarcely probable, that this substance might be identical with phlorizin. The idea of such a possibility arose out of the following considerations :-

1. Glycyphyllin yields phloretin and isodulcite on hydrolysis, whereas phlorizin yields phloretin and a sugar about which some uncertainty has hitherto existed; Hesse maintaining (Miller's Chemistry, 5th Edition, Part III, 582) for reasons to be hereafter mentioned, that it is not identical with ordinary dextrose. Moreover, isodulcite has apparently been more than once mistaken for dextrose. (See Will, Ber., 18, 1312.)

2. Considerable discrepancies are noticeable in the published analysis of phlorizin, so much so that the generally accepted formula of Stas, $\mathrm{C}_{21} \mathrm{H}_{24} \mathrm{O}_{10}$, has been called in question by Loewe (Watts' Dictionary, 8, Part II, p. 1569), who proposes another formula and another equation to represent its hydrolysis. His results, however, as is pointed out (loc. cit.), do not harmonise well with known 
facts. Hugo Schiff also (Ber., 14,308) has drawn attention to the want of agreement in the published analyses of phlorizin.

Under these circumstances, it seemed worth while to re-examine this substance, and to that end a specimen obtained from Trommsdorff was twice recrystallised and then analysed.

\section{Results of Analysis.}

The following are the results in brief of four analyses of the anhydrous substance :-

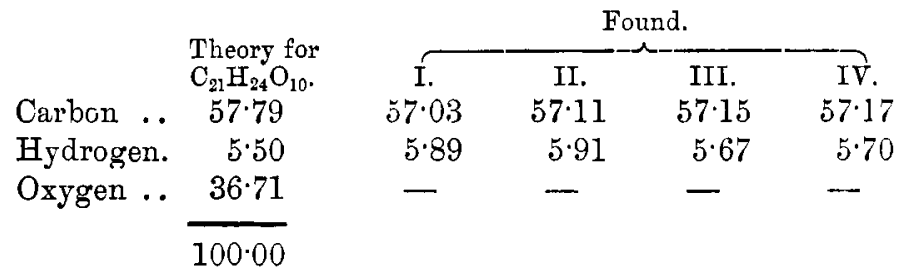

Though these numbers are not very far from those indicated by theory for the above formula, they are scarcely satisfactory. A fresh specimen was therefore prepared by repeated crystallisation. from water. The substance so obtained consisted of perfectly white, well-defined, silky needles; it was analysed without dehydration, having been previously thoroughly air-dried. It contained 7.58 per cent. of water of crystallisation, the theory for 2 mols. $\mathrm{H}_{2} \mathrm{O}$ requiring $7 \cdot 62$ per cent. The following are the results :-

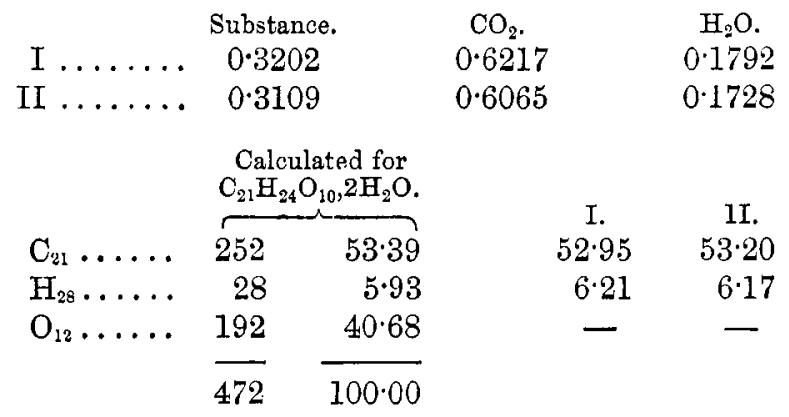

These number are much more satisfactory and confirm the original analyses and formula of Stas.

Decomposition by Ditute Sulphuric Acid.

It being impossible to refer to the original papers here in Adelaide, it has not been possible to ascertain whether any quantitative experi- 
ments hare been made to determine the amounts of phloretin and sugar, respectively, yielded by the hydrolysis of phlorizin. Two experiments were therefore made, the method used being practically the same as that described in the case of glycyphyllin (Trans., 1886, 864), except that the main portion of the phloretin was weighed on a filter-paper of known weight. The results of the titration of the filtrate were of course calculated on the assumption that the sugar is dextrose.

The following were the numbers obtained:-

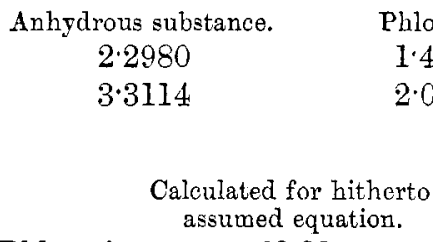

Anhydrous substance.

$2 \cdot 2980$

$3 \cdot 3114$

Calculated for hitherto assumed equation.

Phloretin .... 62.82 per cent.

Dextrose .... 41.28 ,
Phloretin
1.4375
2.0729
Dextrose.

0.9671

$1 \cdot 3514$

These numbers are sufficiently near to those required by the hitherto assumed equation-

$$
\mathrm{C}_{21} \mathrm{H}_{24} \mathrm{O}_{10}+\mathrm{H}_{2} \mathrm{O}=\mathrm{C}_{15} \mathrm{H}_{14} \mathrm{O}_{5}+\mathrm{C}_{6} \mathrm{H}_{12} \mathrm{O}_{6},
$$

to afford strong confirmation of its truth. It may be noted here that the equation by which Loewe (Watts' Dictionary, 8, Part II, p. 1570) represents the decomposition of phorizin, requires 63.5 per cent. phloretin and only 36.4 per cent. dextrose, reckoned on the anhydrous. substance.

\section{Examination of the Sugar obtained.}

The filtrate from the decomposition (by dilute sulphuric acid) of a considerable quantity of phlorizin was neutralised by barium carbonate, filtered and concentrated over a water-bath. A good deal of colouring matter formed. This was removed by repeated treatment with animal charcoal, and the solution finally concentrated to a syrup in a vacuum, over sulphuric acid. On adding a small quantity of water to this syrup, and allowing it to stand for a few days exposed to the air, it gradually became an almost solid mass of minute, perfectly white crystals. These were dried on the filter-pump, washed two or three times with a small quantity of water, and finally spread out to dry in the air.

According to Hesse (Miller's Chemistry, 5th Edition, Part III, p. 582) the sugar from phlorizin differs from dextrose in the following particulars :- 
1. The melting point of the hydrated crystals is $74^{\circ}$, that of dextrose being $82^{\circ}$ according to Hesse (Annalen, 176, 103), or $86^{\circ}$ according to Schmidt (Annalen, 119, 92).

2. It loses only 6.7 per cent. of water at $60-70^{\circ}$, and requires a temperature of $105^{\circ}$ to expel the last traces of water.

3. The specific rotatory power (sodium light) after standing, is $44^{\circ}$, that of dextrose according to various observations ranging from about $53^{\circ}$ to $57.6^{\circ}$ for solutions containing 10 jer cent., or thereabouts, of anhydrous glucose.

With reference to the second point, there seems to be some difference of opinion as to the temperature at which dextrose becomes anhydrous. In the latest edition of Beilstein's Handbuch der organischen Chemie is the following statement: "Krystallisirter Traubenzucker verliert das Krystallwasser unter $100^{\circ}$ unter Scbmelzung und ist bei $110^{\circ}$ wasserfrei," apparently implying that a temperature of $110^{\circ}$ is required to remove the last traces of water.

The sugar under examination melted at $85-86^{\circ}$ when carefully heated in a capillary tube. Heated at $100^{\circ}$, it lost 8.64 per cent. of its water: theory for $1 \mathrm{~mol} \mathrm{H}_{2} \mathrm{O}$ indicating 9.09 per cent. Heated at $102-103^{\circ}$, the loss increased to 9.32 per cent.

A solution was made containing 10.204 grams anhydrous substance in 100 c.c.* This, after standing, gave a rotation of $11.83^{\circ}$ in a tube $200 \mathrm{~mm}$. in length, the observations being made with a Laurent's polarimeter using sodium light. This gives-

$$
[x]_{\mathrm{D}}=\frac{11.83^{\circ}}{2 \times 0.10204}=57.9^{\circ} .
$$

Finally, a solution, when treated with phenylhydrazine hydrochloride in the manner recommended by Fischer (Ber., 17, 579), yielded a yellow, crystalline precipitate exactly corresponding with that described as given by dextrose, and with a melting point 204$205^{\circ}$, when heated in a capillary tube. The facts above adduced leave little doubt that the statements generally made as to the formula and hydrolysis of phlorizin are correct, and that the sugar yielded by it is really dextrose.

* It was intended to make this solution by dissolving a weighed quantity of the crystals in 100 c.c. water, but an accident haring happened to the solution in course of preparation, and no more substance being available, the strength was carefully determined (after dilution) by titration with Febling's solution. 